\title{
Direct CPV in Charm decays at LHCb
}

\author{
Lorenzo Pica ${ }^{a, *}$ \\ ${ }^{a}$ Scuola Normale Superiore \& INFN Pisa, \\ Pisa, Italy \\ E-mail: lorenzo.pica@cern.ch
}

Two searches for direct $C P$ violation in charm decays at $\mathrm{LHCb}$ are presented in this document. The first is a measurement of $\mathcal{A}^{C P}\left(D_{(s)}^{+} \rightarrow h^{+} \pi^{0}\right)$ and $\mathcal{A}^{C P}\left(D_{(s)}^{+} \rightarrow h^{+} \eta\right)$, where $\left(h^{+}=\pi^{+}, K^{+}\right)$. The second is the measurement of $\mathcal{A}^{C P}\left(D^{0} \rightarrow K_{\mathrm{S}}^{0} K_{\mathrm{S}}^{0}\right)$. The latter and five of the results obtained in the former represent the world-best measurement of these quantities up to now.

*** 10th International Workshop on Charm Physics (CHARM2020), ***

*** 31 May - 4 June, 2021 ***

*** Mexico City, Mexico - Online ***

${ }^{*}$ Speaker 


\section{Introduction}

The non-invariance of laws of Nature under Parity $(P)$ and Charge-conjugation $(C)$ operators is known as $C P$ violation $(C P V)$. It can manifest in three different ways: $C P V$ in decay, mixing and interference between decay and mixing.

$C P V$ in the decay is also called direct $C P V$ and it corresponds to a different probability for a process and its $C P$-conjugate to happen:

$$
\mathcal{P}\left(D^{0} \rightarrow f\right) \neq \mathcal{P}\left(\bar{D}^{0} \rightarrow \bar{f}\right)
$$

Discovery of $C P V$ dates back to 1964, when Christenson, Cronin and Fitch confirmed the existence of the $C P$-violating decay of neutral $K_{\mathrm{L}}^{0}$ into pion pairs [1]. Presence of $C P V$ was then verified in the system of $b$-hadrons too, by the $\mathrm{BaBar}$ and Belle collaborations [2,3].

For several decades evidences of $C P V$ were limited to down-type quark hadrons decays, because the size of $C P V$ showed by $c$-hadron decays is small, of the order of $10^{-4}-10^{-3}$ [4]. Therefore, the charm sector provides an excellent environment where to search for influence of New Physics, that could hypothetically couple to up-type quarks only, but at the same time requires the realization of very precise measurements, in order to see any effect. First evidence of $C P V$ in $c$-hadron decays has been found only in 2019 by the LHCb experiment, exploiting the experimentally clean variable $\Delta \mathcal{A}^{C P}=\mathcal{A}^{C P}\left(D^{0} \rightarrow K^{+} K^{-}\right)-\mathcal{A}^{C P}\left(D^{0} \rightarrow \pi^{+} \pi^{-}\right)[5]$.

This measurement represents a milestone in particle physics and opened several questions. The most important one is if there is compatibility between experimental measurement and Standard Model (SM) predictions. In fact, even if the measured value lies in the upper end of theoretical predictions, these are not precise enough to determine if any contribution from Physics beyond the SM is present [4].

In this situation a crucial role is played by further $\mathcal{A}^{C P}$ measurements. They, in fact, represent additional observables that can constrain the comparison with the theory. From these motivations immediately emerges how crucial is to perform additional, high-precision, measurements.

The LHCb detector [6] is a single-arm forward spectrometer, covering the forward pseudorapidity interval $2<\eta<5$. Its main purpose is the study of particles containing bottom and charm hadrons. It is equipped with a warm dipole magnet providing the magnet field able to bend particle trajectories. Its tracking system is made of a vertex detector (VELO) and other tracking stations, placed both upstream and downstream the magnet; these, together, allow a precise measurement of particle momentum. Different particles can be distinguished thanks to the Particle IDentification (PID) system, made of several subdetectors, as ring-imaging Cherenkov and calorimeters. This particular configuration provides $\mathrm{LHCb}$ an high mass resolution and an excellent particle identification, allowing it to make high-precision measurements.

The golden observable exploited to verify the presence of direct $C P V$ is the asymmetry between decay widths of $C P$-conjugate processes. This is defined, for a $D^{0}$ decaying into a final state $f$, as:

$$
\mathcal{A}^{C P}(f)=\frac{\Gamma\left(D^{0} \rightarrow f\right)-\Gamma\left(\bar{D}^{0} \rightarrow \bar{f}\right)}{\Gamma\left(D^{0} \rightarrow f\right)+\Gamma\left(\bar{D}^{0} \rightarrow \bar{f}\right)}
$$

It is not possible to directly extract $\mathcal{A}^{C P}(f)$ from data. The quantity that can be measured is the so-called raw asymmetry, corresponding to the asymmetry between the number of particle and 
anti-particle decays:

$$
\mathcal{A}^{\text {raw }}(f)=\frac{N\left(D^{0} \rightarrow f\right)-N\left(\bar{D}^{0} \rightarrow \bar{f}\right)}{N\left(D^{0} \rightarrow f\right)+N\left(\bar{D}^{0} \rightarrow \bar{f}\right)}=\mathcal{A}^{C P}+\mathcal{A}^{\text {det }}+\mathcal{A}^{\text {prod }}
$$

The raw asymmetry is not only given by the physical asymmetry $\mathcal{A}^{C P}$, but it receives contribution from the detection asymmetry $\mathcal{A}^{\text {det }}$, given by the asymmetrical acceptance of the detector for opposite-sign particles, and the production asymmetry $\mathcal{A}^{\text {prod }}$, result of a different probability of producing matter or antimatter particles in $p p$ interactions. $\mathcal{A}^{\text {det }}$ and $\mathcal{A}^{\text {prod }}$ are usually referred to as nuisance asymmetries.

In order to measure $\mathcal{A}^{C P}, \mathcal{A}^{\text {det }}$ and $\mathcal{A}^{\text {prod }}$ need to be disentangled from $\mathcal{A}^{\text {raw }}$. This is usually done exploiting so-called calibration samples. These are high-statistics samples with a decay chain similar to the analyzed one, for which $\mathcal{A}^{C P}$ is known with a high precision.

In the following sections two of the latest measurements of direct $\mathcal{A}^{C P}$ performed by $\mathrm{LHCb}$ will be presented.

\section{Search for CP violation in $D_{(s)}^{+} \rightarrow h^{+} \pi^{0}$ and $D_{(s)}^{+} \rightarrow h^{+} \eta,\left(h^{+}=\pi^{+}, K^{+}\right)$}

$\mathcal{A}^{C P}$, as defined in Eq. 1, is measured for the two-body $D_{(s)}^{+} \rightarrow h^{+} \pi^{0}$ and $D_{(s)}^{+} \rightarrow h^{+} \eta$ decays, where $h^{+}$can be either a $\pi^{+}$or a $K^{+}$[7]. $\mathcal{A}^{C P}\left(D_{(s)}^{+} \rightarrow h^{+} \pi^{0}\right)$ and $\mathcal{A}^{C P}\left(D_{(s)}^{+} \rightarrow h^{+} \eta\right)$ are measured exploiting data collected by LHCb during Run 1 and Run $2\left(9 \mathrm{fb}^{-1}\right)$ or Run 2 only $\left(6 \mathrm{fb}^{-1}\right)$, respectively.

The study of decays that proceed through Singly Cabibbo Suppressed (SCS) processes is of particular interest. Specifically, $D_{s}^{+} \rightarrow K^{+} \pi^{0}, D^{+} \rightarrow \pi^{+} \eta$ and $D_{s}^{+} \rightarrow K^{+} \eta$ decays allow $C P V$ without the requirement of any second order contribution (usually a penguin diagram), due to the presence of two contributions, with different weak phases, already at tree level. $\mathcal{A}^{C P}$ expected size is $O\left(10^{-4}-10^{-3}\right)$ [8]. $D^{+} \rightarrow \pi^{+} \pi^{0}$ is the other extremely interesting SCS decay. $\mathcal{A}^{C P}\left(D^{0} \rightarrow \pi^{+} \pi^{0}\right)$ is expected to be zero within SM predictions, because of the contributing diagrams and isospin constraints [8], making it an excellent probe for any non-SM contribution.

The reconstruction of a final state only made of one neutral and one charged particle represents a major challenge at LHCb. In fact, it prevents the reconstruction of the $D_{(s)}^{+}$decay vertex, leading to a large combinatorial background contribution to the sample. In order to avoid this condition, the neutral hadron is reconstructed in the $e^{+} e^{-} \gamma$ final state. This can be reached both through the two-body decay $h^{0} \rightarrow \gamma\left(\rightarrow e^{+} e^{-}\right) \gamma$, where one of the photons converts inside the detector material, or through the suppressed three-body Dalitz decay $h^{0} \rightarrow e^{+} e^{-} \gamma$.

A signal candidate is identified from a charged pion or kaon track, associated to a neutral meson candidate; the latter is formed from the combination of a photon and two oppositely charged electron tracks (Bremsstrahlung photons are associated to the reconstructed electron tracks by a specific algorithm).

Selections are applied to reject background, both during trigger selection and offline analysis. Main background component is given by random combination of tracks and photons (purely combinatorial) and real $\pi^{0}$ and charged tracks (real $\pi^{0}$ combinatorial). These are rejected applying selections 
on track quality and displacement of charged tracks, $h^{0}$ and $D_{(s)}^{+}$invariant mass and transverse momentum and $D_{(s)}^{+}$quality vertex. Another background source appears when a $\pi^{ \pm}$track is incorrectly identified as a $K^{ \pm}$and vice versa (misidentification background). This kind of background is rejected applying selections on an MVA-based particle identification variable. Partially reconstructed charm meson decays represent a background, too. These are rejected requiring that the $D_{(s)}^{+}$candidate momentum points toward the Primary Vertex (PV). After the selections two-body $h^{0} \rightarrow \gamma \gamma$ decays represent $86 \%$ of candidates.

$\mathcal{A}^{\text {raw }}$ is measured through a 2D unbinned maximum-likelihood fit to $m\left(e^{+} e^{-} \gamma\right)$ and $m\left(h^{+} h^{0}\right)$, the invariant masses of neutral meson and $D_{(s)}^{+}$, respectively. Sample is split into multiple categories and simultaneously fitted. Categories corresponding to different data taking periods are identified for $D_{(s)}^{+} \rightarrow h^{+} \pi^{0}$ decays, due to different center of mass energy in Run $1(7 \mathrm{TeV}$ and $8 \mathrm{TeV})$ and Run $2(13 \mathrm{TeV})$. Events with zero or one associated Bremsstrahlung photons are put into different categories, while events with two or more are rejected due to poor resolution. Candidates with different charged-hadron type ( $\pi^{ \pm}$or $K^{ \pm}$) are separated, to allow the constraining of misidentification background yield from signal yield of the other category. The last split is based on the charge of the charged hadron.

Probability Density Functions (PDFs) adopted in the fit have different components, to model signal decays and combinatorial, misidentification and partially reconstructed decays. Because of the distributions complex shape, PDFs are modeled on MC data and fine-tuning parameters are included fitting real data, to account for possible data-simulation differences. Fit projections to $D_{(s)}^{+} \rightarrow h^{+} \pi^{0}$ and $D_{(s)}^{+} \rightarrow h^{+} \eta$ candidates are shown in Figure 1 and 2, respectively.

Samples of $D_{(s)}^{+} \rightarrow K_{\mathrm{S}}^{0} h^{+}$decays are exploited as control samples. $\mathcal{A}^{C P}$ is extracted relying on $\mathcal{A}^{\text {det }}$ and $\mathcal{A}^{\text {prod }}$ cancellation, through:

$$
\begin{gathered}
\mathcal{A}^{C P}\left(D_{(s)}^{+} \rightarrow h^{+} h^{0}\right)= \\
=\mathcal{A}^{r a w}\left(D_{(s)}^{+} \rightarrow h^{+} h^{0}\right)-\mathcal{A}^{r a w}\left(D_{(s)}^{+} \rightarrow h^{+} K_{\mathrm{S}}^{0}\right)+\mathcal{A}^{C P}\left(D_{(s)}^{+} \rightarrow h^{+} K_{\mathrm{S}}^{0}\right)+\mathcal{A}_{m i x}\left(K_{\mathrm{S}}^{0}\right)
\end{gathered}
$$

where the first and the second term are extracted from fit to signal and control sample, respectively, the third term comes from previous measurements [9] and the last one accounts for $K_{\mathrm{S}}^{0}$ mixing, regeneration and $C P V$ in the decay.

Before extraction of $\mathcal{A}^{\text {raw }}$, control sample $\mathcal{A}^{\text {det }}$ and $\mathcal{A}^{\text {prod }}$ needs to be equalized to those of the signal. This is firstly done matching trigger and offline selection of the two samples, when possible. Then, in order to ensure a complete equalization of nuisance asymmetries, some control sample distributions are weighted to match signal sample ones. $D_{(s)}^{+}$and $h^{+}$kinematics ( $p$, azimuthal angle and pseudorapidity) are weighted to equalize production and detection asymmetries. Relative fractions of candidates being triggered by different selections are made to match between signal and control sample. $D_{(s)}^{+}$Impact Parameter (IP) ${ }^{1}$ distributions are included in the weighing procedure, as well. This variable, in fact, helps in distinguishing a $D_{(s)}^{+}$candidate that has been produced in the primary $p p$ interaction, from one coming from the decay of a $b$-hadron (secondary decay). The two categories have different $\mathcal{A}^{\text {det }}$ and $\mathcal{A}^{\text {prod }}$, therefore the mixture of the two categories in the samples has to be the same. The sample is weighted separately for Run 1 and Run 2 samples,

${ }^{1}$ Distance of closest approach between the primary vertex and the direction identified by the momentum of the particle. 

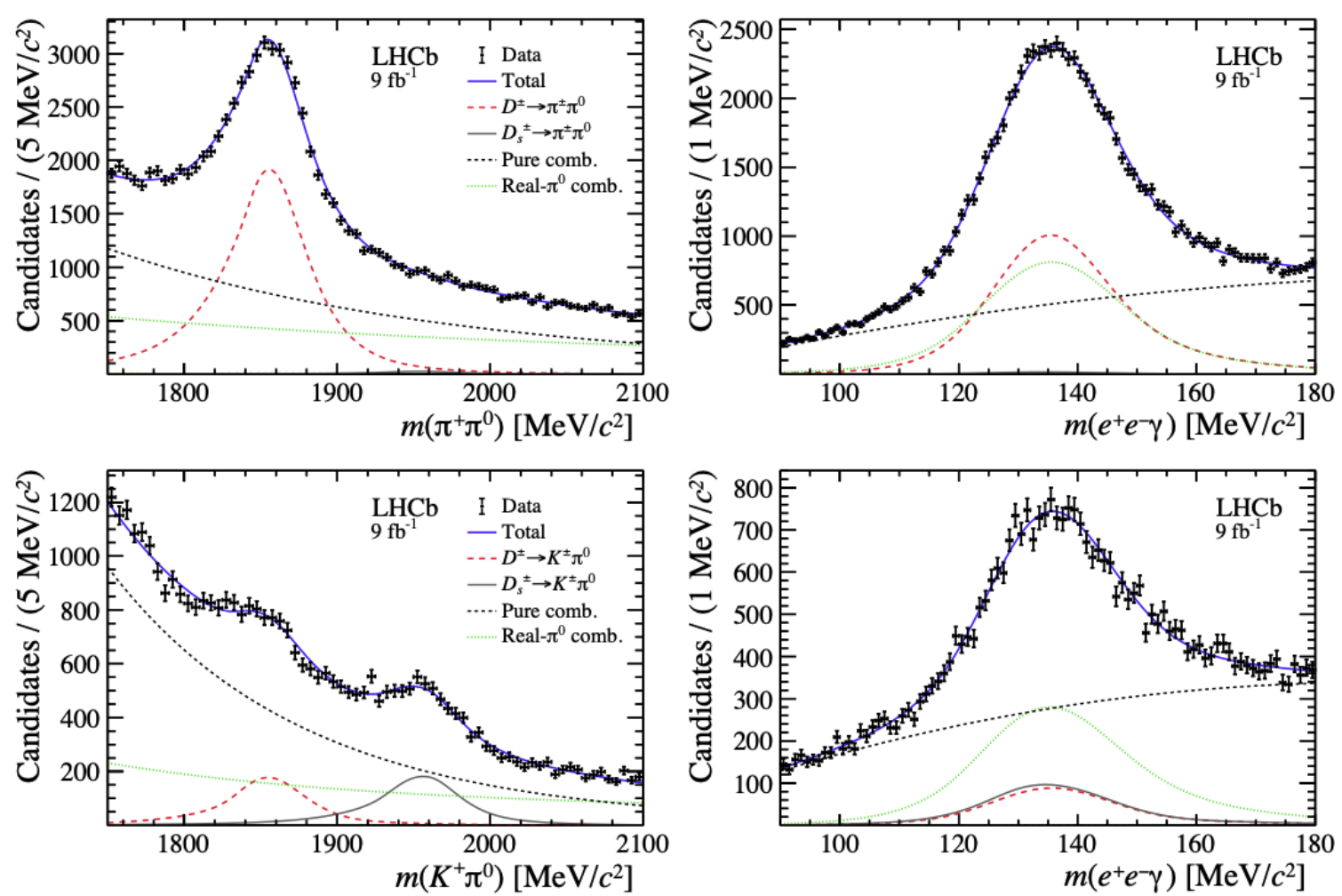

Figure 1: Top and bottom row show $D_{(s)}^{+} \rightarrow \pi^{+} \pi^{0}$ and $D_{(s)}^{+} \rightarrow K^{+} \pi^{0}$, left and right column show $m\left(h^{+} \pi^{0}\right)$ and $m\left(e^{+} e^{-} \gamma\right)$ distributions, respectively.

allowing for differences between different data taking periods to be taken into account. $\mathcal{A}^{\text {raw }}$ and yields are extracted for the control sample through binned maximum-likelihood fits to $m\left(K_{\mathrm{S}}^{0} h^{+}\right)$distributions, as described in [9]. Fit to candidates are shown in Figure 3.

Systematic uncertainties affecting $C P$ asymmetries are estimated. For most of the cases the main contribution comes from the uncertainty on adopted signal and background models. The only exception is the $D_{s}^{+} \rightarrow \pi^{+} \eta$, where the main systematic is due to the statistical uncertainty of the control sample $\left(D_{s}^{+} \rightarrow \pi^{+} K_{\mathrm{S}}^{0}\right)$, since it has the lowest statistics.

Measured values for $\mathcal{A}^{C P}$ extracted exploiting Eq. 2 are

$$
\begin{gathered}
\mathcal{A}^{C P}\left(D^{+} \rightarrow \pi^{+} \pi^{0}\right)=(-1.3 \pm 0.9 \pm 0.6) \%, \\
\mathcal{A}^{C P}\left(D^{+} \rightarrow K^{+} \pi^{0}\right)=(-3.2 \pm 4.7 \pm 2.1) \%, \\
\mathcal{A}^{C P}\left(D^{+} \rightarrow \pi^{+} \eta\right)=(-0.2 \pm 0.8 \pm 0.4) \%, \\
\mathcal{A}^{C P}\left(D^{+} \rightarrow K^{+} \eta\right)=(-6 \pm 10 \pm 4) \%, \\
\mathcal{A}^{C P}\left(D^{+}{ }_{s} \rightarrow K^{+} \pi^{0}\right)=(-0.8 \pm 3.9 \pm 1.2) \%, \\
\mathcal{A}^{C P}\left(D^{+}{ }_{s} \rightarrow \pi^{+} \eta\right)=(0.8 \pm 0.7 \pm 0.5) \%, \\
\mathcal{A}^{C P}\left(D^{+}{ }_{s} \rightarrow K^{+} \eta\right)=(0.9 \pm 3.7 \pm 1.1) \%,
\end{gathered}
$$



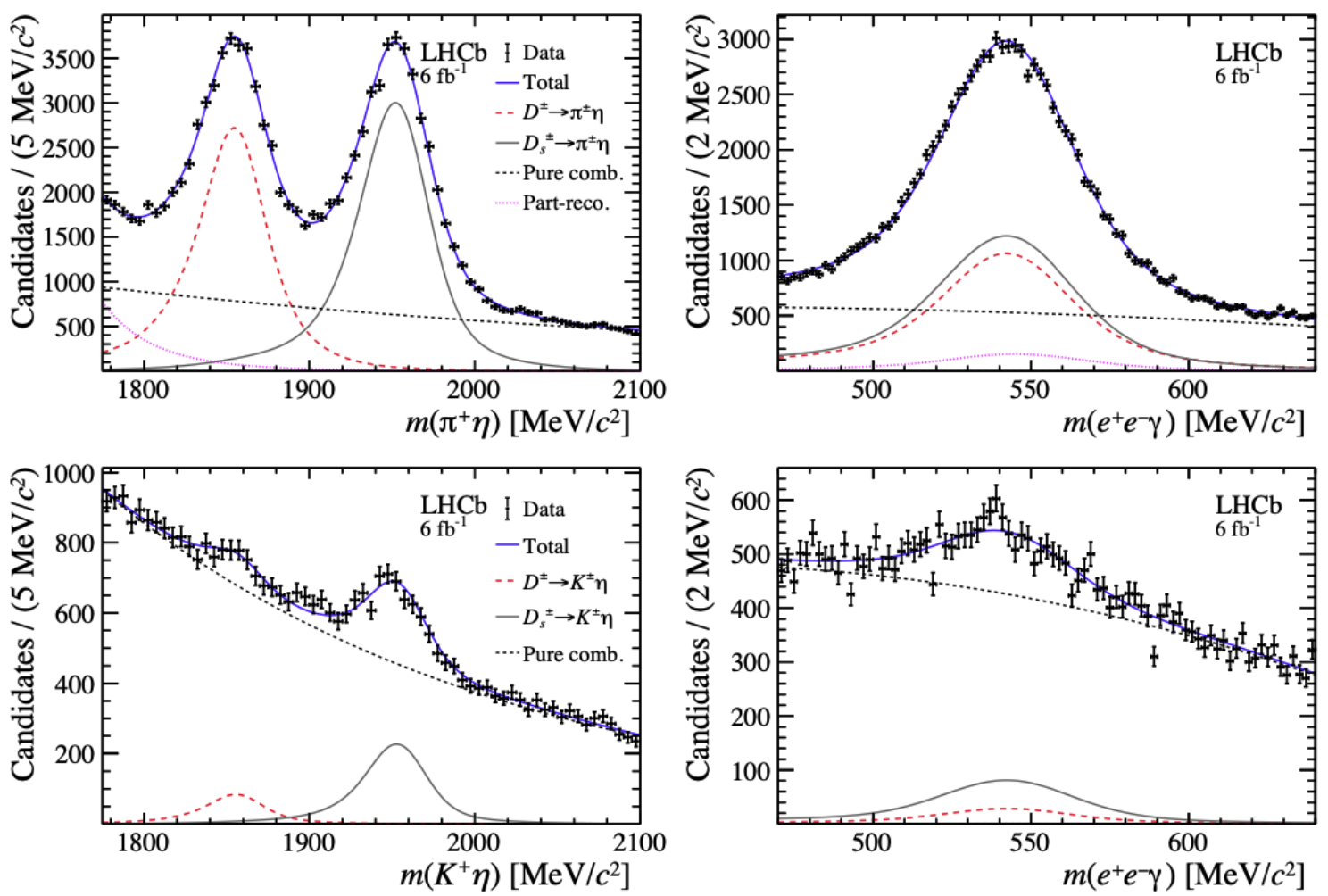

Figure 2: Top and bottom row show $D_{(s)}^{+} \rightarrow \pi^{+} \eta$ and $D_{(s)}^{+} \rightarrow K^{+} \eta$, left and right column show $m\left(h^{+} \eta\right)$ and $m\left(e^{+} e^{-} \gamma\right)$ distributions, respectively.

where the first uncertainty is statistical and the second in systematic. All measured values are compatible with absence of $C P V$. First five results represent the most precise measurements of those quantities to date, a notable result for a measurement performed at a hadron collider.

\section{Measurement of $C P$ asymmetry in $D^{0} \rightarrow K_{\mathrm{S}}^{0} K_{\mathrm{S}}^{0}$ decays at LHCb}

Search for time integrated $C P$ asymmetry is done in $D^{0} \rightarrow K_{\mathrm{S}}^{0} K_{\mathrm{S}}^{0}$ decays, exploiting data collected by LHCb during Run $2\left(6 \mathrm{fb}^{-1}\right)$ [10]. $2015-2016\left(\sim 2 \mathrm{fb}^{-1}\right)$ data are reanalyzed [11] with an improved methodology, achieving a $\sim 30 \%$ sensitivity improvement.

Motivations for this search are based on the possible size of $\mathcal{A}^{C P}\left(D^{0} \rightarrow K_{\mathrm{S}}^{0} K_{\mathrm{S}}^{0}\right)$. It can reach 1\%, according to some of the predictions [12], because of the amplitudes involved in the decay process. Main contributions come from tree-level Exchange (E) and second order Peguin Annihilation (PA) diagrams, reported in Figure 4. The suppression of the tree level diagrams in the $\mathrm{SU}(3)$ limit can make $\mathrm{E}$ and PA diagrams of similar size, leading to a possible $\mathcal{A}^{C P}$ enhancement. This condition makes this decay a possible candidate for an additional measurement of $C P V$ in the charm sector. Additionally, a different mix of amplitudes is involved in this decay with respect to $D^{0} \rightarrow K^{+} K^{-}$and $D^{0} \rightarrow \pi^{+} \pi^{-}$. It can therefore help in improving the theoretical prediction for $\Delta \mathcal{A}^{C P}$.

In order to compute $\mathcal{A}^{C P}\left(D^{0} \rightarrow K_{\mathrm{S}}^{0} K_{\mathrm{S}}^{0}\right)$, it is necessary to tag the flavour of the $D^{0}$. This is done by requiring the $D^{0}$ candidate to come from a strong $D^{*+} \rightarrow D^{0} \pi^{+}$decay (the $C P$-conjugate 

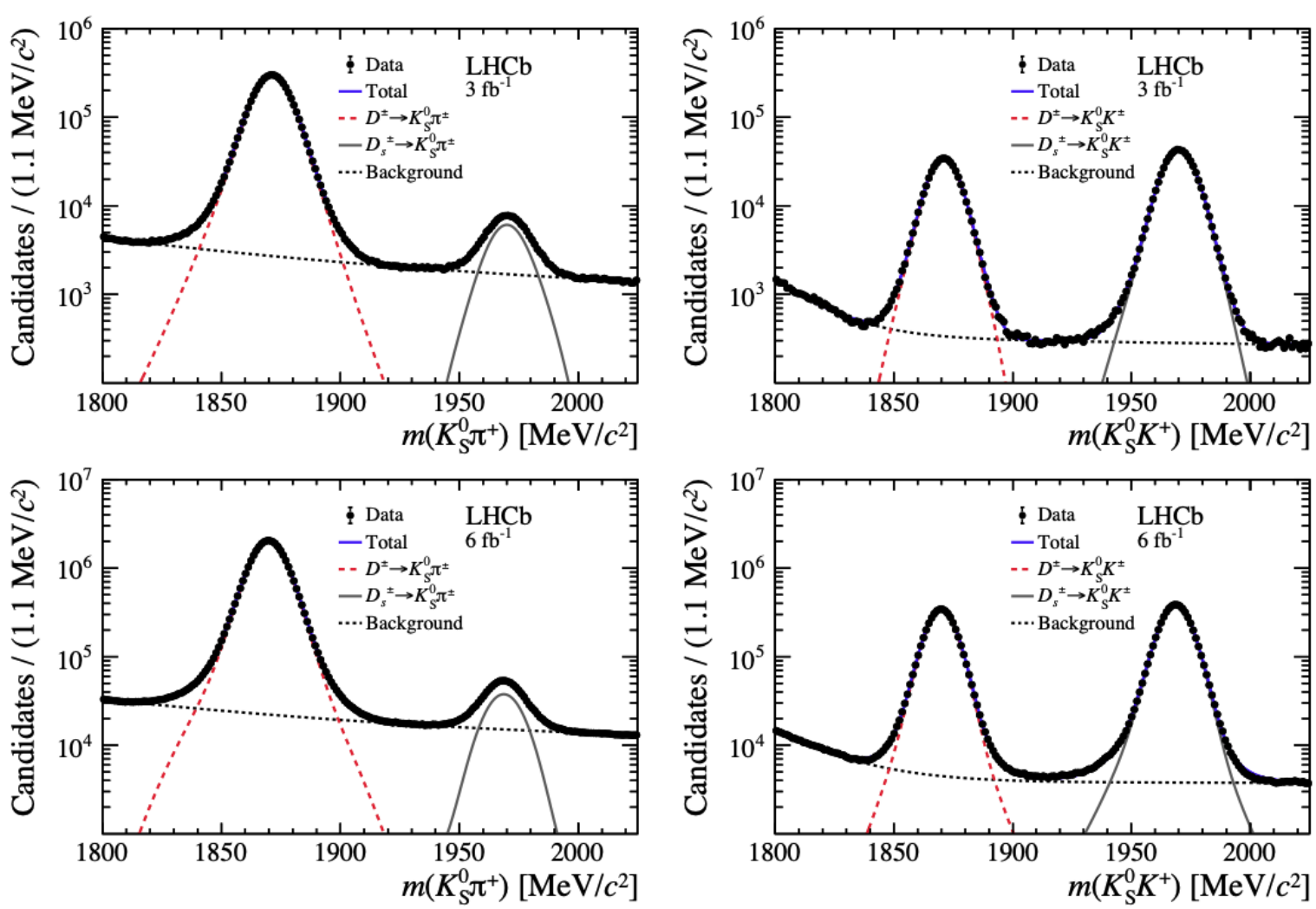

Figure 3: Top and bottom row show Run 1 and Run 2 candidates, left and right column show $m\left(\pi^{+} K_{\mathrm{S}}^{0}\right)$ and $m\left(K^{+} K_{\mathrm{S}}^{0}\right)$ distributions, respectively.

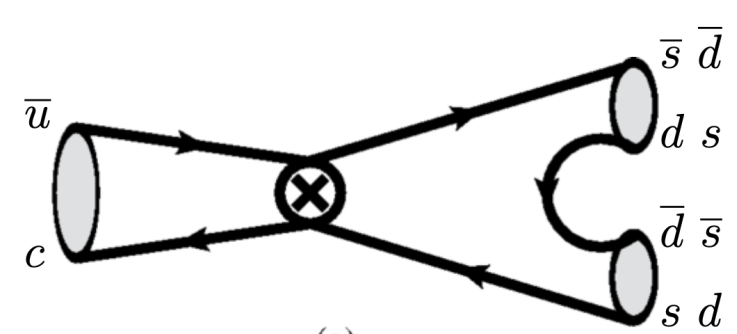

(a)

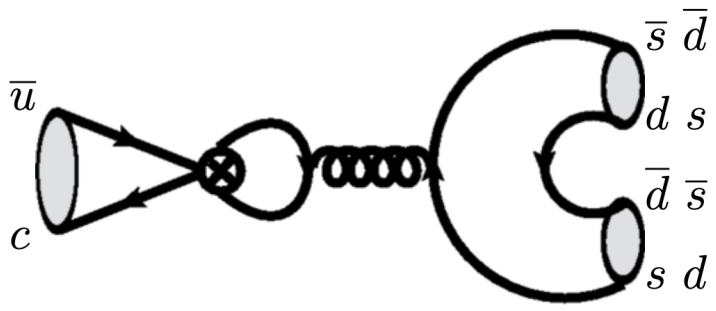

(b)

Figure 4: Exchange (a) and Penguin Annihilation (b) diagrams contributing to the $D^{0} \rightarrow K_{\mathrm{S}}^{0} K_{\mathrm{S}}^{0}$ decay.

process is implied from here); the flavour of the $D^{0}$ can be determined from the sign of the pion coming from the $D^{*}$, usually called tagging-pion $\left(\pi_{\text {tag }}\right)$.

$K_{\mathrm{S}}^{0}$ present in the final state can have large boost and travel for tens or hundreds of centimeters; they can, therefore, be reconstructed both with Long (tracks detected exploiting the entire LHCb tracking system) and Downstream (tracks detected without using the information from the vertex detector) tracks. Due to the presence of two $K_{\mathrm{S}}^{0}$ in the final state, three "categories" are identified and separately analyzed: $\mathrm{LL}, \mathrm{LD}$ and $\mathrm{DD}$, where both $K_{\mathrm{S}}^{0}$ are Long, one $K_{\mathrm{S}}^{0}$ is Long and the other is Downstream or both are Downstream, respectively. This split is necessary because of the different resolution affecting the three channels.

Selections are applied to candidates both during data collection and offline analysis, in order 
to reduce the background contribution.

One of the main background sources is represented by the abundant $D^{0} \rightarrow K_{\mathrm{S}}^{0} \pi^{+} \pi^{-}$decay, where the pion pair is misidentified as a $K_{\mathrm{S}}^{0}$. This can be dangerous if not rejected, since it mimics a signal decay and can lead to a bias in $\mathcal{A}^{C P}$ measurement. Because of this, it is mostly removed before $\mathcal{A}^{C P}$ measurement, applying a cut on $K_{\mathrm{S}}^{0}$ flight distance, and then disentangled from signal in the fit.

Random combination of tracks and candidates is an additional source of background present in the sample. This is made of false $K_{\mathrm{S}}^{0}, D^{0}$ and $D^{*+}$ and represents the most abundant contribution to background. It does not cause any bias, but lowers the $S / B$ ratio. It is partially rejected exploiting a multivariate cut (based on the kNN algorithm [13]) based on tracks kinematics and vertex quality and then disentangled in the fit.

Secondary decays are present in the sample, as well. In previous LHCb measurements they were treated as background, and rejected applying cuts on pointing variables, as the $\operatorname{IP}\left(D^{0}\right)[11,14]$. This strategy causes a significant statistics loss, since $D^{0}$ candidates coming from the primary $p p$ interaction (prompt) and secondary decays can not be clearly distinguished. This is due to the resolution on the position of the $D^{0}$ vertex decay, shown in Figure 5. In this condition, in order to effectively reject most of secondary decays, tight selections have to be applied, determining the discard of a significant part of prompt decays, too. In this analysis, to avoid this side effect, it has

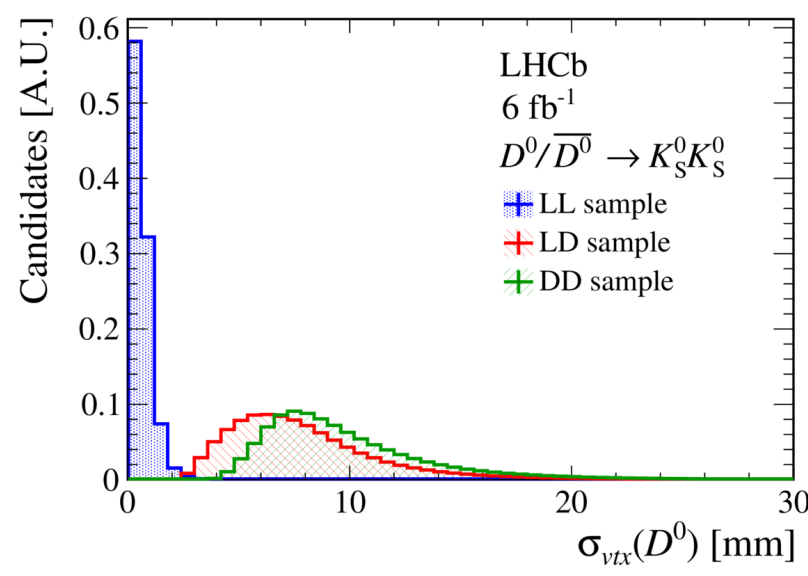

Figure 5: Distributions of the uncertainty on the $D^{0}$ vertex position for $D^{0} \rightarrow K_{\mathrm{S}}^{0} K_{\mathrm{S}}^{0}$ decays, separately presented for LL, LD and DD samples.

been decided not to follow the strategy of previous measurements. Here secondary decays are not rejected, but included in the signal sample.

To avoid the presence of biases in $\mathcal{A}^{C P}$ measurement, the relative fraction of prompt and secondary decays needs to be the same between signal and control samples. This is obtained adopting as control channel a large sample of $D^{0} \rightarrow K^{+} K^{-}$, collected without any cut on variables related to the $D^{0}$ vertex, equalizing control channel selections to the ones of the signal sample and avoiding the application of any cut on variables with different resolution in the two samples. Doing this, any applied cut has the same effect on the prompt-secondary mixture in the two samples, ensuring the absence of any bias on $\mathcal{A}^{C P}$.

The method adopted to cancel nuisance asymmetries in this analysis is different from the one 
usually exploited in LHCb. Here, in fact, it is done weighting each signal candidate with a weight that is:

$$
w^{ \pm}(\mathbf{p})=\frac{n_{K^{+} K^{-}}^{+}(\mathbf{p})+n_{K^{+} K^{-}}^{-}(\mathbf{p})}{2 n_{K^{+} K^{-}}^{ \pm}(\mathbf{p})} \cdot\left[1 \pm \mathcal{A}^{C P}\left(K^{+} K^{-}\right)\right]
$$

Where $\mathbf{p}$ is the $D^{0}$ three-momentum, $n_{K^{+} K^{-}}(\mathbf{p})$ is the calibration sample local density around a given value of $\mathbf{p}$ and $\mathcal{A}^{C P}\left(K^{+} K^{-}\right)$is the measured value for $\mathcal{A}^{C P}$ in that channel, taken from [15]. Adopting this weight, $\mathcal{A}^{\text {det }}$ and $\mathcal{A}^{\text {prod }}$ affecting $D^{0} \rightarrow K^{+} K^{-}$are exploited to automatically cancel out $D^{0} \rightarrow K_{\mathrm{S}}^{0} K_{\mathrm{S}}^{0}$ sample nuisance asymmetries, in an event by event approach. After this procedure, $\mathcal{A}^{C P}$ can be directly extracted from the fit, in the same way as $\mathcal{A}^{\text {raw }}$ was previously extracted.

Asymmetries and yields of signal and background components are extracted through a 3D maximum-likelihood fit to the mass difference $\Delta m=m\left(D^{0} \pi_{t a g}\right)-m\left(K_{\mathrm{S}}^{0} K_{\mathrm{S}}^{0}\right)$ and the two $K_{\mathrm{S}}^{0}$ candidates invariant mass $m\left(\pi^{+} \pi^{-}\right)$distributions.

In order to maximize the sensitivity of the measurement, the sample is split into several subsamples. The first split is between different channels: LL, LD and DD; this is done because of the different resolutions of the three. The sample is also split according to different data taking periods: 2015 - 2016 and 2017 - 2018; different trigger selections have been used in these two periods. Different subsamples are identified depending on their purity level, defined according to kNN algorithm output. Finally, events for which the $D^{*+}$ decay vertex is compatible or not with the PV are kept separate; this allows the mass resolution to be improved in case of compatibility between the vertices, forcing them to coincide when fitting the decay chain.

Distributions and fit projections for some representative subsamples are shown in Figure 6.

Several systematic sources are taken into account and estimated. Main systematic effect is given by the uncertainty on models adopted to fit mass distributions, being them empirical.

Result obtained combining measurements extracted from the fit to all the subasamples is

$$
\mathcal{A}^{C P}\left(D^{0} \rightarrow K_{\mathrm{S}}^{0} K_{\mathrm{S}}^{0}\right)=(-3.1 \pm 1.2 \pm 0.4 \pm 0.2) \%
$$

where the first uncertainty is statistical, the second is systematic and the third comes from the knowledge on $\mathcal{A}^{C P}\left(D^{0} \rightarrow K^{+} K^{-}\right)$.

This represents the most precise measurement of $\mathcal{A}^{C P}\left(D^{0} \rightarrow K_{\mathrm{S}}^{0} K_{\mathrm{S}}^{0}\right)$ to date and it is compatible with the hypothesis of $C P$ conservation within $2.4 \sigma$. The new world average, obtained from the combination with previous measurements $[14,16,17]$, is equal to

$$
\mathcal{A}^{C P}\left(D^{0} \rightarrow K_{\mathrm{S}}^{0} K_{\mathrm{S}}^{0}\right)_{W A}=(-1.9 \pm 1.0) \%
$$

and it approaches for the first time the predictions upper limit, set to $1 \%$ [12].

\section{Conclusions}

Huge samples of $D^{0}$ decays collected by LHCb during Run 1 and Run 2 allowed the achievement of many high precision measurements. This made possible the first observation of $C P V$ in charm decays, in 2019. Now efforts of several experiments are focused on the realization of additional measurement, that can potentially confirm this result and help in shedding light on this new field of 

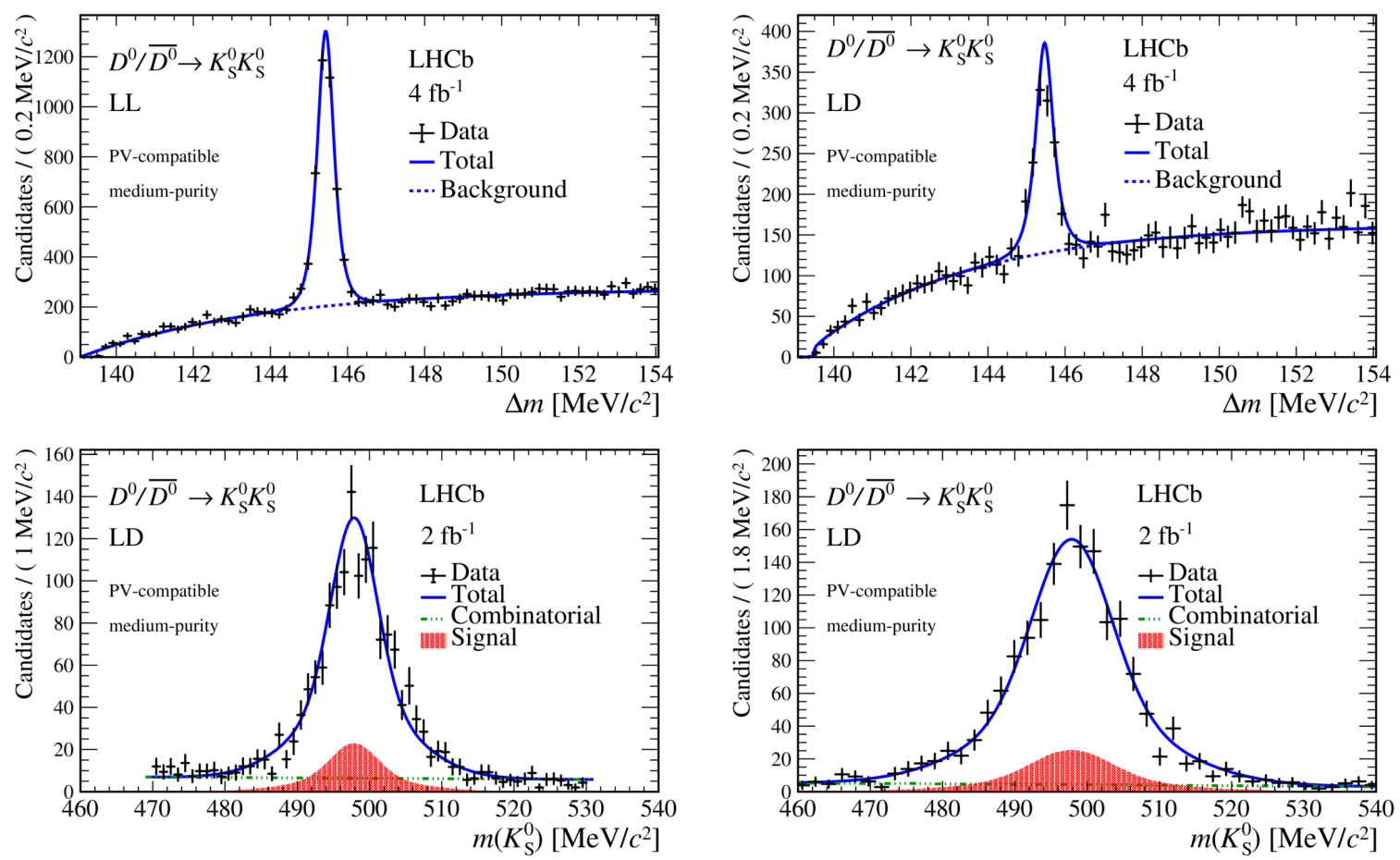

Figure 6: In the top row distributions and fit projections for $\Delta m$ are shown, for the LL, PV-compatible, medium-purity, 2017 - 2018 (left) and LD, PV-compatible, medium purity, 2017 - 2018 (right) subsamples. In the bottom row distributions and fit projections for Long (left) and Downstream (right) $m\left(\pi^{+} \pi^{-}\right)$are shown, for the LD, PV-compatible, medium-purity, 2015 - 2016 sample.

$C P V$. LHCb is playing a major role in chasing this result, producing many world-best measurements, as the two presented in this document. It is of major significance to notice that this is happening also for decays with neutral mesons in the final state $\left(\pi^{0}, \eta\right.$ and $\left.K_{\mathrm{S}}^{0}\right)$. Despite these results, no confirmation of $C P V$ in charm decays has been found yet.

The search will continue with data that will be collected from 2022 by LHCb with the Run 3 of LHC. LHCb is strenuously working in order to be able collect even larger samples than the already available ones. This will be made possible by the higher luminosity at which the experiment will work, and by the upgraded detector and trigger system, that will allow to collect more data per $\mathrm{fb}^{-1}$ than it was before.

\section{References}

[1] J.H. Christenson, J.W. Cronin, V.L. Fitch and R. Turlay, Evidence for the $2 \pi$ Decay of the $K_{2}^{0}$ Meson, Phys. Rev. Lett. 13 (1964) 138.

[2] BABAR COLLABORATION collaboration, Measurement of CP-violating asymmetries in $b^{0}$ decays to CP eigenstates, Phys. Rev. Lett. 86 (2001) 2515.

[3] A. Abashian, K. Abe, K. Abe, I. Adachi, B.S. Ahn, H. Aihara et al., Measurement of the $C P$ violation parameter $\sin 2 \varphi_{1}$ in $b_{d}^{0}$ meson decays, Phys. Rev. Lett. 86 (2001) 2509. 
[4] Y. Grossman, A.L. Kagan and Y. Nir, New physics and CP violation in singly Cabibbo suppressed D decays, Phys. Rev. D 75 (2007) 036008 [hep-ph/0609178].

[5] LHCв collaboration, Observation of CP Violation in Charm Decays, Phys. Rev. Lett. 122 (2019) 211803 [1903.08726].

[6] LHCв collaboration, The LHCb Detector at the LHC, JINST 3 (2008) S08005.

[7] R. Aaij, C. Abellán Beteta, T. Ackernley, B. Adeva, M. Adinolfi, H. Afsharnia et al., Search for cp violation in $D_{(s)}^{+} \rightarrow h^{+} \pi^{0}$ and $D_{(s)}^{+} \rightarrow h^{+} \eta$ decays, Journal of High Energy Physics 2021 (2021).

[8] H.-n. Li, C.-D. Lu and F.-S. Yu, Branching ratios and direct $c p$ asymmetries in $d \rightarrow p p$ decays, Phys. Rev. D 86 (2012) 036012.

[9] LHCв Collaboration collaboration, Search for cp violation in $D_{s}^{+} \rightarrow K_{S}^{0} \pi^{+}, D^{+} \rightarrow K_{S}^{0} K^{+}$, and $D^{+} \rightarrow \phi \pi^{+}$decays, Phys. Rev. Lett. 122 (2019) 191803.

[10] LHCв Collaboration collaboration, Measurement of cp asymmetry in $D^{0} \rightarrow K_{\mathrm{s}}^{0} K_{\mathrm{s}}^{0}$ decays, Phys. Rev. D 104 (2021) L031102.

[11] LHCB COLLABORATION collaboration, Measurement of the time-integrated CP asymmetry in $D^{0} \rightarrow K_{\mathrm{S}}^{0} K_{\mathrm{S}}^{0}$ decays, JHEP 11 (2018) 048 LHCb-PAPER-2018-012 CERN-EP-2019-065, [1806.01642].

[12] U. Nierste and S. Schacht, $C P$ Violation in $D^{0} \rightarrow K_{S} K_{S}$, Phys. Rev. D92 (2015) 054036 [1508.00074].

[13] J.Friedman, T.Hastie and R.Tibshirani, The Elements of Statistical Learning, Springer Series in Statistics (2001).

[14] LHCB collaboration, Measurement of the time-integrated $C P$ asymmetry in $D^{0} \rightarrow K_{S}^{0} K_{S}^{0}$ decays, JHEP 10 (2015) 055 [1508.06087].

[15] LHCв collaboration, Measurement of CP asymmetry in $D^{0} \rightarrow K^{-} K^{+}$decays, Phys. Lett. B 767 (2017) 177 [1610.09476].

[16] CLEO collaboration, Search for $C P$ violation in $D^{0} \rightarrow K_{S}^{0} \pi^{0}$ and $D^{0} \rightarrow \pi^{0} \pi^{0}$ and $D^{0} \rightarrow K_{S}^{0} K_{S}^{0}$ decays, Phys. Rev. D63 (2001) 071101 [hep-ex/0012054].

[17] N. Dash et al., Search for CP Violation and Measurement of the Branching Fraction in the Decay $D^{0} \rightarrow K_{S}^{0} K_{S}^{0}$, Phys. Rev. Lett. 119 (2017) 171801 [1705.05966]. 